\title{
The Value of Tumor Treating Fields in Glioblastoma
}

\author{
Chaochao Zhang, ${ }^{1}$ Jianyang Du, ${ }^{2}$ Weidong Xu, ${ }^{1}$ Haiyan Huang, ${ }^{1}$ Li Gao ${ }^{3}$ \\ Department of Neurosurgery, First Hospital of Jilin University, Changchun, China \\ Department of Neurosurgery, ${ }^{2}$ The Second Affiliated Hospital, Harbin Medical University, Harbin, China \\ Department of Outpatient, ${ }^{3}$ First Hospital of Jilin University, Changchun, China
}

Glioblastoma (GBM) is one of the most common tumors of the central nervous system, which is the most lethal brain cancer. GBM treatment is based primarily on surgical resection, combined with radiotherapy and chemotherapy. Despite the positive treatment, progression free survival and overall survival were not significantly prolonged because GBM almost always recurs. We are always looking forward to some new and effective treatments. In recent years, a novel treatment method called tumor treating fields (TTFields) for cancer treatment has been proposed. TTFields devices were approved by the Food and Drug Administration (FDA) for adjuvant treatment of recurrent and newly diagnosed GBMs in 2011 and 2015, respectively. This became the first breakthrough treatment for GBM in the past 10 years after the FDA approved bevacizumab for patients with relapsed GBM in 2009. This paper summarized the research results of TTFields in recent years and elaborated the mechanism of action of TTFields on GBM, including cell and animal experimental research, clinical application and social benefits.

Key Words : Tumor treating fields · Glioma · Glioblastoma · Mitosis · Apoptosis.

\section{CURRENT STATUS OF GLIOBLASTOMA (GBM) TREATMENT}

Glioma originates from glial cells and is the most common intracranial tumor. At present, the World Health Organization classifies gliomas into grades I to IV. Grades I and II were low-grade gliomas, and grade III and IV were high-grade glio$\mathrm{mas}^{5)}$. GBM is defined as grade IV, which accounts for about half of primary brain malignant tumors, and has the charac- teristics of high incidence, high recurrence rate, high mortality and low cure rate ${ }^{20)}$. At present, the main treatment is the classical Stupp protocol, which is the largest surgical excision within the safe range. After operation, conventional fractionated radiotherapy (60 Gy/30-33 F) concurrent with daily temozolomide (TMZ) chemotherapy, plus adjuvant chemotherapy for TMZ for 6-12 courses are also required ${ }^{11,25)}$. There are also PCV programs : procarbazine, lomustine, vincristine, 8 weeks for a course ${ }^{29)}$. The drugs used in the treatment of glio-

- Received : November 6, 2019 •Revised : December 30, 2019 •Accepted : January 23, 2020

- Address for reprints : Haiyan Huang

Department of Neurosurgery, First Hospital of Jilin University, 71 Xinmin Avenue, Changchun 130021, China

Tel : +86-13578967878, Fax : +86-88782222, E-mail : huanghy@jlu.edu.cn, ORCID : https://orcid.org/0000-0003-1082-2692

\section{Li Gao}

Department of Outpatient, First Hospital of Jilin University, 71 Xinmin Avenue, Changchun 130021, China

Tel : +86-18843106325, Fax : +86-88782291, E-mail : 18843106325@163.com, ORCID : https://orcid.org/0000-0002-5945-8310

This is an Open Access article distributed under the terms of the Creative Commons Attribution Non-Commercial License (http://creativecommons.org/licenses/by-nc/4.0) which permits unrestricted non-commercial use, distribution, and reproduction in any medium, provided the original work is properly cited. 
ma include carmustine, irinotecan, etoposide, cisplatin, carboplatin, cyclophosphamide and so on. Despite aggressive multimodal therapy, the prognosis of patients with GBM remains poor. Based on the current treatment methods, the treatment effect of newly diagnosed GBM is still poor, and it is still very easy to recur after conventional surgery plus radiotherapy and chemotherapy. Until now, there is not any unified standard treatment for recurrent high-grade glioma. Therefore, Individualized treatment should be formulated according to the location of the tumor, the physical condition of the patient, previous treatment methods and efficacy. So far, BEV or $\mathrm{TMZ}$ is recommended in combination with radiotherapy and/or a new treatment. So tumor treating fields (TTFields) it came into being.

\section{WORKING PRINCIPLE OF TTFIELDS}

The electric field is divided into (DC) electrical field and alternating current (AC) electric field. AC has been proved to have a wide range of biological effects and has been applied in medical fields such as scientific research, disease diagnosis and treatment. AC electric field with different frequencies and intensities has different biological effects. DC or low-frequency AC electric fields promote bone growth and fracture healing through membrane depolarization ${ }^{3)}$; high-frequency AC electric fields cause electrolyte polarization and produce ther- mal effects on tissues eventually, which have been applied in diathermy or ablation technology ${ }^{28)}$. In the past, the frequency of electric field alternation at the intermediate frequency (10$1000 \mathrm{kHz}$ ) was too fast to induce cells ${ }^{1)}$. In an important breakthrough paper, Kirson et al. ${ }^{16)}$ reported that an AC field with a low intensity $(1-3 \mathrm{~V} / \mathrm{cm})$ intermediate frequency (100$300 \mathrm{kHz}$ ) significantly inhibited the growth rate of various mammalian tumor cells, which is called TTFeilds. This is a wearable device called Optune, and the first-generation Optune system (known as the NovoTTF-100-A system, CE Mark 2007) consists of two main components : an electric field generator and an array of insulated transducers (Fig. 1). TTFeilds exerts a role in inhibiting tumor growth by applying an insulating electrode to the human body. Current studies have shown that electric field therapy has the advantages of safety, less adverse reactions. It is recommended for the treatment of newly diagnosed GBM and recurrent $\mathrm{GBM}^{24,26)}$.

\section{BIOLOGICAL RESPONSE OF TTFIELDS TO CELLS}

The cells contain charged particles (such as proteins and DNA), including unipolarity charged polar particles and double-charged dipoles, which are subject to Coulomb forces in the electric field. In the uneven AC electric field, the alternating Coulomb force magnitude or direction of all charged particles is different, and the Coulomb force's clearance effect is

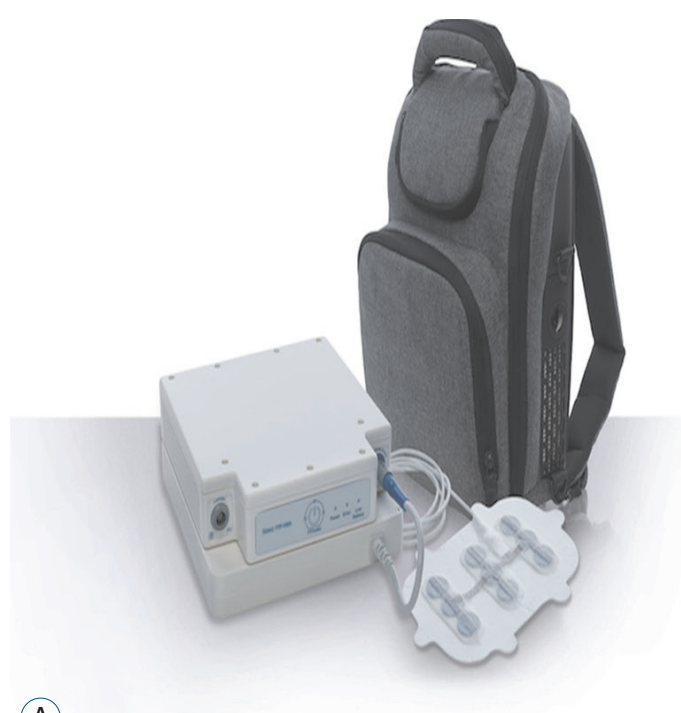

(A)

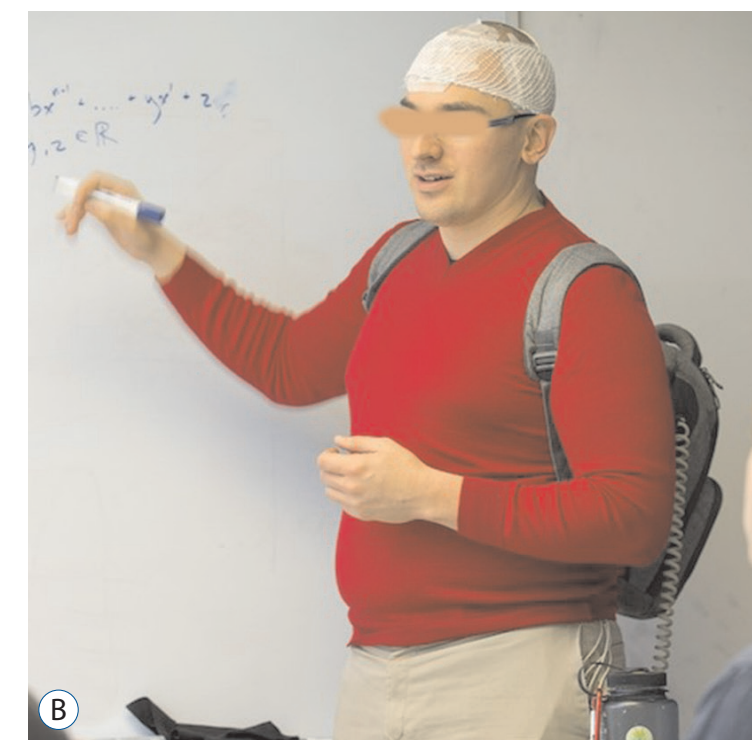

Fig. 1. A :TTFields devices. B : A patient wearing TTFields devices. TTFields : treatment method called tumor treating fields. 
not zero, then the charged particles will be under the action of the electric field force.

The direction of high electric field density is called dielectric electrophoresis ${ }^{4,8)}$. In non-dividing cells, the electric field formed by the addition of an alternating electric field in the cell is substantially uniform. Therefore, the electric field force of the charged particles in the alternating electric field (the frequency is high enough, such as TTFields) is zero, and no motion is generated. TTFields have little effect on normal resting cells and only work on dividing tumor cells ${ }^{15}$.

Tumor cell mitosis is impaired in two aspects : 1) when the tumor cell mitosis ends in the late stage, the electric field forms a uniform electric field in a single cell, and the charged microtubule is pulled by a uniform electric field, which cannot form the normal spindle microtubule structure in the tumor cells, and further lead to the subsequent abnormal division finally. And 2) when the tumor cells enter the late stage of mitosis, two sub-sub-cells are gradually formed, and after the electric field is applied, an intracellular non-uniform alternating electric field is formed. At this time, under the guidance of the electric field, the charged substance in the cells concentrated to the cleavage furrow, which causes the cell membrane pressure to rise, eventually leading to cell blistering and rupture (Fig. 2) ${ }^{9,16,27)}$. In particular, cell division can be destroyed only when the long axis of the cell cleavage ditch is parallel to

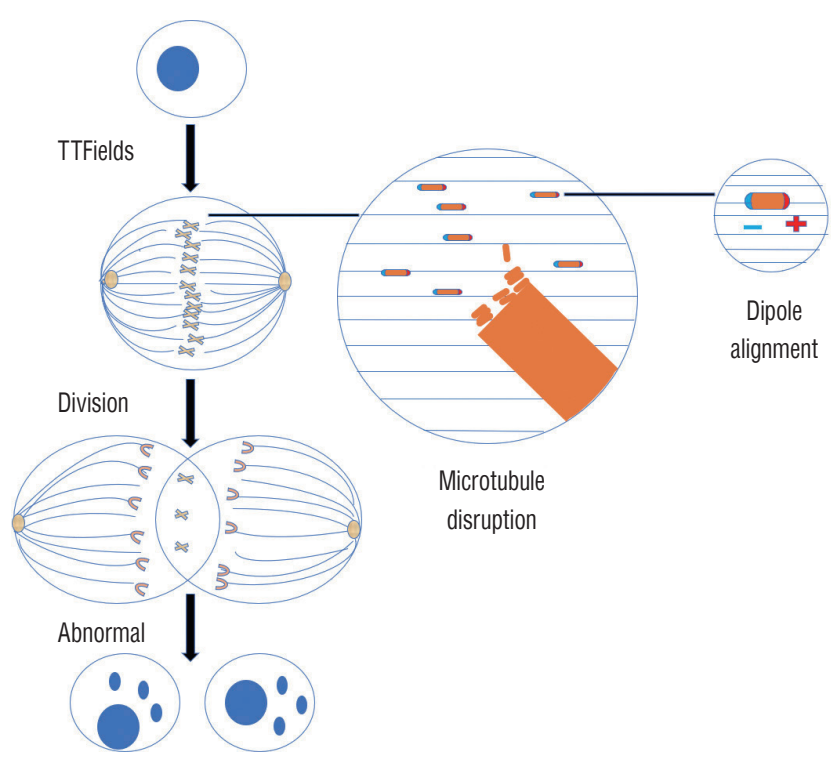

Fig. 2. The alteration of the mitotic spindle during mitosis by TTFields that results in death. TTFields : treatment method called tumor treating fields. the direction of the electric field, and normal nerve cells are considered unaffected because of slow division. TTFields arrest tumor cell mitosis for a long time in the interphase, prolong the mitotic cycle of tumor cells, and even cause cell fragmentation and inhibit tumor cell proliferation.

\section{PROGRESS OF TTFIELDS ON CELL EXPERIMEN- TAL RESEARCH}

Cytological experiments of TTFields show that the intermediate frequency alternating electric field has an inhibitory effect on tumor cell proliferation, and its inhibition is related to the time, frequency, intensity, direction and cell type ${ }^{16)}$. In addition, the sensitivity of different cells to electric field intensity is different, the optimal inhibition breast cancer cells) is 150 kHz, F-98 cells (rat glioma cells) and U-118, U-87 (human glioma cells) is $200 \mathrm{kHz}$. Based on this, the main frequency of the subsequent application of electric field therapy equipment to glioma treatment was established ${ }^{15)}$. TTFields induced autophagy by blocking the Akt2/miR29b axis in GBM cells ${ }^{14}$. TTFields are also capable of impeding the migration and invasion of glioma cells ${ }^{12,22}$. Furthermore, sorafenib significantly improved the therapeutic efficiency of TTFields by inhibiting tumor cell survival, apoptosis, cell cycle regulation, autophagy, inhibiting tumor cell invasion and angiogenesis ${ }^{13)}$. So far, sensitive parameters of various cell lines have been found, for example, A2780 ovarian cancer is $200 \mathrm{kHz}$, lung cancer A549, pancreatic cancer AsPC-1, cervical cancer HeLa, mesothelioma MSTO-211H, and breast cancer MCF-7 are $150 \mathrm{kHz}^{7}$. The effect of TTFields is related to the direction of electric field and splitting axis. When the two directions are parallel, the effect is the greatest, and when the two directions are vertical, the effect is the worst. A study showed that the effect of alternating two perpendicular electric fields was 20\% higher than that of unidirectional electric fields on B16F1 cells and F98 cells $^{12,16)}$.

\section{PROGRESS OF TTFIELDS ON ANIMAL EXPERI- MENTAL RESEARCH}

In 2004, Kirson et al. ${ }^{16)}$ described the role of TTFields mediated by implanted electrode patches in animal models of mel- 
anoma for the first time. After 3-6 days of treatment, the tumors in the treatment group were dramatically smaller than those in the control group. Histopathological analysis of treated tumors showed a large number of apoptotic and necrotic tumor cells. However, no damage was detected in the surrounding tissues. Later, a study demonstrated the effectiveness of TTFields mediated by external electrode patches in the treatment of intracranial GBM in rats ${ }^{15)}$. Interestingly, it was found that $100 \mathrm{kHz}$ medium frequency alternating electric field had no significant effect on the growth of tumors, while $200 \mathrm{kHz}$ TTFields caused significant inhibition on the growth of tumors $^{15)}$. After 6 days of treatment, with an ideal frequency of $200 \mathrm{kHz}$, the maximum diameter of tumors of the treatment group was nearly half that of the control group. Unidirectional TTFields had little effect, which did not reach the statistical significance. However, increasing the number of TTFields directions caused statistically significant inhibition of tumor growth, reaching $42.6 \%$ and $53.4 \%$ for two and three directions positioned at $45-90^{\circ}$ to each other, respectively. In addition, after being applied to healthy animals for one month, no treatment-related side effects and toxicity were observed. A recent study found that xenografts treated with TTFields progressed at a slower rate and the tumor volume was small by bearing subcutaneous human U87 tumors. In addition, in nude mice bearing xenografts of U373 GBM cells, combination therapy of sorafenib and TTFields significantly inhibited tumor growth and prolonged animal survival ${ }^{13)}$.

\section{PROGRESS OF TTFIELDS ON HUMAN GBM CLINICAL TRIAL}

Kirson et al. ${ }^{15)}$ described the safety and efficacy of TTField (NovoTTF-100A device) treatment for 10 patients with recurrent GBM for the first time. TTFields with the optimal frequency of $200 \mathrm{kHz}$ and an intensity of 1-2 V/cm were employed in the trial. Patients were treated for an average of 18 hours a day until disease progression or for a maximum of 18 months. Progression free survival (PFS) and overall survival (OS) were 26.1 and 62.2 weeks respectively, which were almost twice as much as the control group. Some side effects sometimes occurred during the treatment, and the most common adverse event was contact dermatitis caused by the electrode gel in nine patients, which could be well treated with steroid cream. In addition, Salzberg et al. ${ }^{21)}$ used an electric field of $100-200 \mathrm{kHz}$ to treat several other cancer patients and metastatic patients, which ultimately showed potential efficacy.

From 2006 to 2009, in a phase III clinical trial, 237 patients with recurrent GBM from multi-centre were randomly DC TTFields monotherapy (120 patients) and optimal chemotherapy group (117 patients) according to the ratio of $1: 1^{26)}$. This is the first randomized controlled trial to evaluate a novel treatment modality for recurrent GBM, transfer electric field and chemotherapy. This is the first randomized controlled trial evaluating an entirely novel cancer treatment modality delivering electric fields. The OS was 6.6 months and 6.0 months (hazard ratio [HR], $0.86 ; p=0.27$ ), the 1 -year survival rate was the same $20 \%$ and $20 \%$, and the median PFS was 2.2 months and 2.1 months $(p=0.16), 6$-month PFS rate was $21.4 \%$ and $15.1 \%(p=0.13)$, and imaging efficiencies were $14 \%$ and $9.6 \%$ $(p=0.19)$, respectively in TTFields and control patients. However, O6-methylguanine-DNA methyltransferase (MGMT) gene, an important predictive factor for the efficacy of TMZ in the treatment of GBM, was not evaluated in this study.

Light-moderate contact dermatitis occurs in 16\% of patients treated with TTFields, and only 3\% showed severe events. Although the trial failed to show a significant improvement in survival rate, it can dramatically improve the quality of life and become a treatment highlight. Given this, the first-generation TTFields device, known at the time as the NovoTTF100A System (renamed Optune), was approved by FDA for treatment of recurrent GBM in 2011.

Subsequently, there was an experimental adjuvant treatment with NovoTTF-100A, celecoxib and BEV illustrating the possibility of improving survival with end-stage recurrent $\mathrm{GBM}^{30)}$.

Another retrospective study showed that the OS and PFS of 18 GBM patients receiving TMZ, BEV, and irinotecan in combination with TTFields were 18.9 and 10.7 months, respective$\mathrm{ly}^{18)}$. It can be seen that the treatment plan has great potential for GBM patients. Hypertension (38.9\%) were the most common side effects without unexpected severe toxicities in this study ${ }^{18)}$.

In newly diagnosed GBM patients, TTFields combined with TMZ had a PFS of 155 weeks and OS of over 39 months, whereas the control group received maintenance TMZ alone only had PFS and OS of 31 weeks and 14.7 months, respective$1 y^{17)}$. In a phase III clinical trial, 695 patients with newly diag- 
nosed GBM who had completed standard surgery and concurrent chemoradiotherapy were randomized $2: 1$ to the TTFields plus TMZ treatment group and the TMZ treatment group alone. The median OS of the former group and the latter were 20.9 months and 16.0 months (HR, 0.63 ; $p<0.01$ ), respectively, while the median PFS was 6.7 months and 4.0 months $(\mathrm{HR}, 0.63 ; p<0.01)^{24)}$. It can be seen that the use of TTFields combined with TMZ can significantly prolong OS and PFS in patients who have completed concurrent chemoradiotherapy. Then the secondary analysis of this trail also consistent with the previous interim analysis, but MGMT promoter methylation was more prevalent in the control group ${ }^{23)}$.

According to a recent meta analysis ${ }^{19)}$, the median OS was increased in patients treated with TTFields with recurrent GBM (weighted mean difference [WMD], 2.55; $p<0.00001$ ) and newly diagnosed GBM (WMD, 7.48; $p<0.00001$ ). In addi- tion, patients who received the TTField modality presented increased OS, survival at after 1 and 2 years, PFS, along with PFS at 6 months. Subgroup analysis indicates patients older than 65 years and those with MGMT promoter methylation tumors (regardless of age) have a greater increase in OS.

We searched the ClinicalTrials.gov website and found that there are still 10 ongoing clinical studies on TTFields treatment of GBM (Table 1).

\section{SIDE EFFECTS AND VALUE OF TTFIELDS TREATMENT}

Compared with conventional chemotherapy, in the TTFields group, the frequency and intensity of adverse reactions such as anorexia, diarrhea, constipation, nausea and vomiting,

Table 1. Ongoing trials using TTFields for GBM as of August 2019

\begin{tabular}{|c|c|c|c|c|c|c|}
\hline NCT Number & Condition & Intervention & Characteristic & Outcome measure & Population & Date \\
\hline NCT02743078 & Recurrent GBM & $\begin{array}{l}\text { Bevacizumab } \\
\text { TTFields }\end{array}$ & Phase 2 & OS6, OS, PFS, ORR, AE & 3 & $\begin{array}{l}\text { April 12, } 2017 \text { to } \\
\text { August } 2020\end{array}$ \\
\hline NCT02663271 & Recurrent GBM & $\begin{array}{l}\text { Bevacizumab } \\
\text { Optune }\end{array}$ & Phase 2 & $P F S, A E, K P S$ & 18 & $\begin{array}{l}\text { August } 2016 \text { to } \\
\text { March } 2020\end{array}$ \\
\hline NCT02903069 & Newly diagnosed GBM & $\begin{array}{l}\text { MRZ } \\
\text { TMZ } \\
\text { RT } \\
\text { Optune }\end{array}$ & Phase 1 & OS, PFS, AE & 73 & $\begin{array}{c}\text { February 10, } 2016 \text { to } \\
\text { August 31, } 2019\end{array}$ \\
\hline NCT03405792 & Newly diagnosed GBM & $\begin{array}{l}\text { TMZ } \\
\text { Optune } \\
\text { Pembrolizumab }\end{array}$ & Phase 2 & OS, PFS, AE & 29 & $\begin{array}{c}\text { February 23, } 2018 \text { to } \\
\text { February } 2023\end{array}$ \\
\hline NCT03223103 & Newly diagnosed GBM & $\begin{array}{l}\text { Poly-ICLC } \\
\text { TTFields } \\
\text { Peptides }\end{array}$ & Phase 1 & PFS, OS, ORR & 20 & $\begin{array}{c}\text { March 1, } 2018 \text { to } \\
\text { May 22, } 2021\end{array}$ \\
\hline NCT03705351 & Newly diagnosed GBM & $\begin{array}{l}\text { TTFields } \\
\text { TMZ } \\
\text { RT }\end{array}$ & Phase 1 & PFS6, PFS24, ORR, AE & 30 & $\begin{array}{c}\text { April 1, } 2019 \text { to } \\
\text { April 1, } 2025\end{array}$ \\
\hline NCT02893137 & Recurrent GBM & $\begin{array}{l}\text { Optune } \\
\text { Craniectomy }\end{array}$ & Phase 1 & PFS, PFS6, OS, AE, ORR & 15 & $\begin{array}{c}\text { October } 2016 \text { to } \\
\text { October } 2019\end{array}$ \\
\hline NCT03687034 & GBM & $\begin{array}{l}\text { TMZ } \\
\text { Optune }\end{array}$ & Phase 1 & AE, PFS & 21 & $\begin{array}{l}\text { June } 2019 \text { to } \\
\text { September } 2019\end{array}$ \\
\hline NCT01894061 & Recurrent GBM & $\begin{array}{l}\text { Bevacizumab } \\
\text { NovoTTF-I00A }\end{array}$ & Phase 2 & $\begin{array}{l}\text { PFS, ORR, OS, TTP, } \\
\text { NCF, QOL }\end{array}$ & 40 & $\begin{array}{l}\text { June 12, } 2013 \text { to } \\
\text { December } 2019\end{array}$ \\
\hline NCT02343549 & Newly diagnosed GBM & $\begin{array}{l}\text { Bevacizumab, } \\
\text { NovoTTF-100A, TMZ }\end{array}$ & Phase 2 & PFS12 & 46 & $\begin{array}{l}\text { January } 2015 \text { to } \\
\text { June } 2021\end{array}$ \\
\hline
\end{tabular}

TTFields : treatment method called tumor treating fields, GBM : glioblastoma, OS6 : overall survival at 6 months, OS : overall survival, PFS : progression free survival, ORR : objective response rate, AE : adverse event, KPS : Karnofsky Performance scale, MRZ : marizomib, TMZ : temozolomide, RT : radiotherapy, ICLC : lysine carboxymethylcellulose, ORR : objective response rate, PFS6 : progression free survival at 6 months, PFS24 : progression free survival at 24 months, TTP : time-to-progression, NCF : neurocognitive function, QOL : quality of life, PFS12 : progression free survival at 12 months 
pain and fatigue were significantly reduced ${ }^{13,14)}$. The patient had milder blood system damage, lower gastrointestinal reactions, and no increased incidence of epilepsy. In summary, the most common adverse reaction of TTFields treatment is skin side effects caused by prolonged contact between the electrode sheet and the skin, including irritant contact dermatitis, allergic dermatitis, ulcers, skin infections or pustules, which is also the main reason for the decrease of patient compliance. Therefore, strengthening prevention, symptomatic treatment and reducing the incidence are the top priorities. Fortunately, adverse skin reactions can be prevented by appropriate measures. Preventive measures include health education for patients and caregivers, proper skin preparation, infection prevention, avoidance of contact with scar areas, and proper placement of electrodes. Therapeutic measures mainly include drug treatment and suspension of TTFields treatment. Drug treatment mainly includes the application of topical glucocorticoids and the application of topical antibiotics. If local symptoms of dermatitis occur, local application of glucocorticoids is recommended, when the epithelial barrier is destroyed (smashed) or the appearance of infection has been found, antibiotics are recommended. The choice of antibiotics depends on the type of scalp bacteria, and mupirocin ointment and polymyxin B ointment are often used. For refractory severe skin reactions, it is recommended to suspend TTFields for 2-7 days. This showed that all skin reactions are reversible.

TTFields undoubtedly prolonged survival, the researchers also evaluated the quality of life of patients. There was no significant difference in overall health and social ability between the TTFields group and the TMZ group, but in terms of cognition, etiquette, and affective function, the former group was significantly better than the latter.

\section{TTFIELDS EQUIPMENT AND SOCIAL BENEFITS}

The first-generation Optune system, the NovoTTF-100-A system, and the second-generation Optune system, the NovoTTF-200-A system, weigh approximately $2.7 \mathrm{~kg}$ and $1.2 \mathrm{~kg}$, respectively. Therefore, the second-generation of devices is expected to be more user-friendly, in terms of convenience, compliance, and manageability in treating TTFields patients with $\mathrm{GBM}^{6}$. Importantly, compliance (average monthly use) is also closely related to better clinical outcomes. But things are not as simple as we think. Before wearing the device, patients must shave their heads to attach scalp electrodes. The caregiver should teach the patient to operate the device independently, but compliance decreases outside the hospital, making it hard to guarantee that patients carry batteries for more than 18 hours a day.

There are other problems. Older or weaker patients may find it more difficult to wear and transport the device. In fact, the price of Optune is not affordable for all patients. For example, in the United States, the price of treatment without commercial insurance is $\$ 21000 /$ person/month, $\$ 150368 /$ person/year of life, $\$ 198032 /$ person/quality adjusted life year, ${ }^{2,10)}$. A study evaluated the cost-effectiveness of TTFields and TMZ for newly diagnosed GBM from the perspective of the US healthcare system ${ }^{10)}$. Treatment with TTFields and TMZ was expected to give rise to a mean increase in survival of 1.25 life years and 0.96 quality-adjusted life years (QALYs) compared to treatment with TMZ alone. The incremental total cost was $\$ 188637$ and the incremental cost-effectiveness ratio was $\$ 150452$ per life year gained and \$197336 per QALY gained. The model was most sensitive to changes in the cost of TTFields treatment. Therefore, TTFields treatment can be seen as cost-effective within the reported range of willingness-to-pay thresholds in the USA.

\section{CONCLUSION}

TTFields is a therapeutic method that exerts anti-tumor effect by inhibiting the mitosis of tumor cells. It plays an important role in autophagy, apoptosis, cell proliferation, angiogenesis and migration of glioma cells. Many studies have shown that TTFields is safe and effective for the treatment of newly diagnosed GBM and high-grade recurrent glioma including GBM. It may have many mild to moderate side effects, but they are reversible. In addition, it costs a lot, but at the same time, the benefits are worthwhile. There are still about ten clinical trials under way. We believe that more and more largescale TTFields alone or in combination with other treatments can provide the best treatment for glioma patients to maximize patient outcomes and the incidence of adverse reactions, and bring more benefits to glioma patients. which is the direction of our efforts. 


\section{CONFLICTS OF INTEREST}

No potential conflict of interest relevant to this article was reported.

\section{INFORMED CONSENT}

This type of study does not require informed consent.

\section{AUTHOR CONTRIBUTIONS}

\author{
Conceptualization : CZ \\ Data curation : JD \\ Formal analysis : JD \\ Funding acquisition : WX \\ Methodology: WX \\ Project administration : LG \\ Visualization : LG \\ Writing - original draft : $\mathrm{CZ}$ \\ Writing - review \& editing : HH, LG
}

\section{ORCID}

Chaochao Zhang https://orcid.org/0000-0002-3761-6381

Jianyang $\mathrm{Du} \quad$ https://orcid.org/0000-0001-9682-4183

Weidong Xu

Haiyan Huang

https://orcid.org/0000-0002-2984-5686

Li Gao

https://orcid.org/0000-0003-1082-2692

https://orcid.org/0000-0002-5945-8310

\section{References}

1. Barnes FS, Greenebaum B : Handbook of Biological Effects of Electromagnetic Fields, ed 3. Boca Raton : CRC Press, 2006

2. Bernard-Arnoux F, Lamure M, Ducray F, Aulagner G, Honnorat J, Armoiry $X$ : The cost-effectiveness of tumor-treating fields therapy in patients with newly diagnosed glioblastoma. Neuro Oncol 18 : 1129-1136, 2016

3. Ciombor DM, Aaron RK : The role of electrical stimulation in bone repair. Foot Ankle Clin 10 : 579-593, vii, 2005

4. Clague DS, Wheeler EK : Dielectrophoretic manipulation of macromolecules: the electric field. Phys Rev E Stat Nonlin Soft Matter Phys 64(2 Pt 2) : 026605, 2001
5. DeWitt JC, Mock A, Louis DN : The 2016 WHO classification of central nervous system tumors: what neurologists need to know. Curr Opin Neurol 30 : 643-649, 2017

6. Fabian D, Guillermo Prieto Eibl MDP, Alnahhas I, Sebastian N, Giglio P, Puduvalli $\mathrm{V}$, et al. : Treatment of glioblastoma (GBM) with the addition of tumor-treating fields (TTF): a review. Cancers (Basel) $11: 174$, 2019

7. Giladi M, Schneiderman RS, Voloshin T, Porat $Y$, Munster M, Blat R, et al. : Mitotic spindle disruption by alternating electric fields leads to improper chromosome segregation and mitotic catastrophe in cancer cells. Sci Rep 5 : 18046, 2015

8. Gonzalez CF, Remcho VT : Harnessing dielectric forces for separations of cells, fine particles and macromolecules. J Chromatogr A 1079 : 59 68,2005

9. Gutin PH, Wong ET : Noninvasive application of alternating electric fields in glioblastoma: a fourth cancer treatment modality. Am Soc Clin Oncol Educ Book 32 : 126-131, 2012

10. Guzauskas GF, Pollom EL, Stieber VW, Wang BCM, Garrison LP Jr : Tumor treating fields and maintenance temozolomide for newly-diagnosed glioblastoma: a cost-effectiveness study. J Med Econ 22 : 1006-1013, 2019

11. Hegi ME, Diserens AC, Gorlia T, Hamou MF, de Tribolet N, Weller M, et al. : MGMT gene silencing and benefit from temozolomide in glioblastoma. N Engl J Med 352 : 997-1003, 2005

12. Hondroulis E, Melnick SJ, Zhang X, Wu ZZ, Li CZ : Electrical field manipulation of cancer cell behavior monitored by whole cell biosensing device. Biomed Microdevices 15 : 657-663, 2013

13. Jo Y, Kim EH, Sai S, Kim JS, Cho JM, Kim H, et al. : Functional biological activity of sorafenib as a tumor-treating field sensitizer for glioblastoma therapy. Int J Mol Sci $19: 3684,2018$

14. Kim EH, Jo Y, Sai S, Park MJ, Kim JY, Kim JS, et al. : Tumor-treating fields induce autophagy by blocking the Akt2/miR29b axis in glioblastoma cells. Oncogene $38: 6630-6646,2019$

15 Kirson ED, Dbalý V, Tovarys F, Vymazal J, Soustiel JF, Itzhaki A, et al. : Alternating electric fields arrest cell proliferation in animal tumor models and human brain tumors. Proc Natl Acad Sci U S A 104 : 1015210157, 2007

16. Kirson ED, Gurvich Z, Schneiderman R, Dekel E, Itzhaki A, Wasserman Y, et al. : Disruption of cancer cell replication by alternating electric fields. Cancer Res 64 : 3288-3295, 2004

17. Kirson ED, Schneiderman RS, Dbalý V, Tovarys F, Vymazal J, Itzhaki A, et al. : Chemotherapeutic treatment efficacy and sensitivity are increased by adjuvant alternating electric fields (TTFields). BMC Med Phys $9: 1$, 2009

18. Lu G, Rao M, Zhu P, Liang B, El-Nazer RT, Fonkem E, et al. : Triple-drug therapy with bevacizumab, irinotecan, and temozolomide plus tumor treating fields for recurrent glioblastoma: a retrospective study. Front Neurol $10: 42,2019$

19. Magouliotis DE, Asprodini EK, Svokos KA, Tasiopoulou VS, Svokos AA, Toms SA : Tumor-treating fields as a fourth treating modality for glioblastoma: a meta-analysis. Acta Neurochir (Wien) 160 : 1167-1174, 
2018

20. Ostrom QT, Gittleman H, Farah P, Ondracek A, Chen Y, Wolinsky Y, et al. : CBTRUS statistical report: primary brain and central nervous system tumors diagnosed in the United States in 2006-2010. Neuro Oncol 15 Suppl 2 : ii1-ii56, 2013

21. Salzberg M, Kirson E, Palti Y, Rochlitz C : A pilot study with very lowintensity, intermediate-frequency electric fields in patients with locally advanced and/or metastatic solid tumors. Onkologie 31 : 362-365, 2008

22. Silginer $M$, Weller $M$, Stupp $R$, Roth $P$ : Biological activity of tumortreating fields in preclinical glioma models. Cell Death Dis 8 : e2753, 2017

23. Stupp R, Taillibert S, Kanner A, Read W, Steinberg D, Lhermitte B, et al. : Effect of tumor-treating fields plus maintenance temozolomide vs maintenance temozolomide alone on survival in patients with glioblastoma: a randomized clinical trial. JAMA 318 : 2306-2316, 2017

24. Stupp R, Taillibert $S$, Kanner AA, Kesari S, Steinberg DM, Toms SA, et al. : Maintenance therapy with tumor-treating fields plus temozolomide vs temozolomide alone for glioblastoma: a randomized clinical trial. JAMA 314 : 2535-2543, 2015

25. Stupp R, Tonn JC, Brada M, Pentheroudakis G; ESMO Guidelines Working Group : High-grade malignant glioma: ESMO clinical practice guide- lines for diagnosis, treatment and follow-up. Ann Oncol 21 Suppl 5 : v190-v193, 2010

26. Stupp R, Wong ET, Kanner AA, Steinberg D, Engelhard H, Heidecke V, et al. : NovoTTF-100A versus physician's choice chemotherapy in recurrent glioblastoma: a randomised phase III trial of a novel treatment modality. Eur J Cancer 48 : 2192-2202, 2012

27. Swanson KD, Lok E, Wong ET : An overview of alternating electric fields therapy (NovoTTF Therapy) for the treatment of malignant glioma. Curr Neurol Neurosci Rep $16: 8,2016$

28. Tuszynski JA, Wenger C, Friesen DE, Preto J : An overview of sub-cellular mechanisms involved in the action of TTFields. Int J Environ Res Public Health $13: 1128,2016$

29. van den Bent MJ, Carpentier AF, Brandes AA, Sanson M, Taphoorn MJ, Bernsen $\mathrm{HJ}$, et al. : Adjuvant procarbazine, lomustine, and vincristine improves progression-free survival but not overall survival in newly diagnosed anaplastic oligodendrogliomas and oligoastrocytomas: a randomized European Organisation for Research and Treatment of Cancer phase III trial. J Clin Oncol 24 : 2715-2722, 2006

30. Wong ET, Lok E, Swanson KD : Clinical benefit in recurrent glioblastoma from adjuvant NovoTTF-100A and TCCC after temozolomide and bevacizumab failure: a preliminary observation. Cancer Med 4 : 383-391, 2015 